\title{
Current status of hypothermia as a treatment modality
}

Michael M. Todd MD

I NTEREST in hypothermia is longstanding; early laboratory experiments were done more than 70 years ago and the effects of cooling on whole body and brain metabolic rates have been known since the early 1940's. Only in 1943 did Temple Fay report his initial experience with the use of hypothermia in head injured patients but in spite of exceptionally positive results ( $100 \%$ recovery!), the neurosurgical community showed little interest. In contrast, the growth of cardiac surgery - first closed and then open - created a demand for techniques that made procedures easier and safer, and by 1950, the use of hypothermia was widespread. Even after the introduction of cardiopulmonary bypass, hypothermia remained a routine component of cardiac surgery until very recently - although more for "cardiac preservation" than for anything else.

Outside of cardiac surgery, the clinical use of hypothermia was sporadic. For example, in the 1960's there was a transient surge in the use of cooling (often with some kind of circulatory arrest) during the early days of intracranial aneurysm surgery. This disappeared largely due to the introduction of better operative tools (and the microscope). However, in the late 1980's, a laboratory observation changed the landscape. Prior to the late 1980's, it had been assumed that since $\mathrm{CMRO}_{2}$ fell in a curvilinear fashion with cooling, the degree of central nervous system (CNS) protection was directly proportional to temperature. However, in the late 1980's, Busto and colleagues in Miami demonstrated that even small reductions in temperature could have dramatic protective benefits in animal models of cerebral ischemia. The importance of such work was the fact that protection could be achieved with temperatures that were relatively easy to achieve.

This triggered a marked resurgence of interest in clinical hypothermia and within a few years, hypothermia had, for example, become routine in neurovascular operating rooms. In 1992, a survey of neuroanesthesiologists by Rosemary Craen and
Adrian Gelb suggested that nearly a third cooled their intracranial aneurysm patients. A recent survey in Great Britain indicated that this number had risen to nearly $60 \%$. This enthusiasm has persisted - a 2003 British survey suggests that as many as $60 \%$ of neuroanesthesiologists used hypothermia during vascular surgery - in spite of: a) a lack of any controlled data indicating the benefits of hypothermia in humans; and b) a body of evidence suggesting that hypothermia may result in increased risks for wound infection, pneumonia, hemorrhage, and myocardial ischemia.

With this background, I will present and discuss the existing data concerning the clinical use of mild hypothermia in five areas:

I. Cardiac surgery (not circulatory arrest);

II. Head trauma;

III. Stroke;

IV. Cardiac arrest;

V. Intracranial vascular surgery.

A more detailed discussion of the individual studies will be presented at the meeting; I will only summarize the material here.

\section{Cardiac surgery}

While moderate hypothermia (temperatures between 28 and $32^{\circ} \mathrm{C}$ ) was long used primarily for the purpose of myocardial preservation, there was a general assumption that it had beneficial CNS effects as well. This was based on existing animal data and on the well known protective effects of deep hypothermia during circulatory arrest. The shift away from systemic hypothermia triggered the first formal clinical trials examining the neurologic impact of cooling during bypass. There have now been several such trials - and the general consensus, as summarized by the Cochrane group, is that there are no advantages to cooling.

\section{Head trauma}

Soon after the Busto studies appeared, laboratory work appeared demonstrating the beneficial effects of

From the Department of Anesthesia, University of Iowa College of Medicine, Iowa City, Iowa, USA.

Address correspondence to: Dr. Michael M. Todd, Department of Anesthesia, University of Iowa College of Medicine, 200 Hawkins Drive 6546 JCP, Iowa City, IA 52242-1009, USA. Phone: 319-356-4601; Fax: 319-353-6817; E-mail: anesthesiology@uiowa.edu 
cooling applied after head injury in rats. Initial human studies suggested that hypothermia could be useful in controlling intracranial pressure. This quickly led to a small clinical trial, which seemed to show a benefit, which led in turn to a large definitive trial. However, that large trial convincingly demonstrated that hypothermia did not improve outcome and several smaller trials in the last two years seem to confirm this. One major problem is logistical; it is very difficult to cool patients quickly after their injuries. There is, hence, a new trial now in progress, which is enrolling ONLY patients who are cold on arrival in the emergency room, randomizing them to either continued cold or to rewarming. If the cold group has a better outcome, it will serve as an impetus to the development of more efficient methods for rapidly cooling patients, perhaps even in the field or during transport.

\section{Stroke}

In spite of a great deal of publicity, no controlled trials of cooling in stroke have been published at the time of this writing.

\section{Cardiac arrest}

The animal work that triggered our modern interest in hypothermia involved models of global cerebral ischemia - the same form of insult encountered in cardiac arrests. Multiple animal studies confirmed both the protective effects of cooling (started before arrest) and its therapeutic benefit (started after resuscitation). Last year, the results of two human studies were reported, both demonstrating a rather strong beneficial effect of post-resuscitative cooling in patients who had suffered from ventricular fibrillation (VFib). While the work is only directly applicable to a small fraction of cardiac arrest victims, the American Heart Association has now recommended that cooling be used in successfully resuscitated VFib patients.

\section{Intracranial vascular surgery}

As noted above, hypothermia remains popular among neuroanesthesiologists. This popularity, combined with the risks noted above, led to the development of a large clinical trial entitled "Intraoperative Hypothermia for Aneurysm Surgery Trial (IHAST)." This was a prospective randomized trial of 1,000 patients undergoing open aneurysm surgery following subarachnoid hemorrhage, with half of the patients being cooled to a target of $33^{\circ} \mathrm{C}$ during surgery. Enrollment and follow-up was completed in September of 2003 and results will be presented in February 2004. These results and their implications will be presented.

\section{Selected references}

Fay T. Observations of generalized refrigeration in cases of severe cerebral trauma. Assoc Res Nerv Ment Dis Proc 1943; 24: 611-9.

Bigelow R. Hypothermia. Its possible role in cardiac surgery. Ann Surgery 1950; 132: 849.

Busto R, Dietrich WD, Globus MY, Valdes I, Scheinberg P, Ginsberg MD. Small differences in intraischemic brain temperature critically determine the extent of ischemic neuronal injury. J Cereb Blood Flow Metab 1987; 7: 729-38.

Pemberton PL, Dinsmore J. The use of hypothermia as a method of neuroprotection during neurosurgical procedures and after traumatic brain injury: a survey of clinical practices in Great Britain and Ireland. Anaesthesia 2003; 58: 371-3.

Frank SM, Fleisher LA, Breslow MJ, et al. Perioperative maintenance of normothermia reduces the indicence of morbid cardiac events. A randomized clinical trial. JAMA 1997; 277: 1127-34.

Kurz A, Sessler DI, Lenhardt R, for the Study of Wound Infection and Temperature Group. Perioperative normothermia to reduce the incidence of surgical-wound infection and shorten hospitalization. $\mathrm{N}$ Engl J Med 1996; 334: 1209-15.

The Warm Heart Investigators. Randomized trial of normothermic versus hypothermic coronary bypass surgery. Lancet 1994; 343: 559-63.

Engelman RM, Pleet $A B$, Rousou JA, et al. Influence of cardiopulmonary bypass perfusion temperature on neurologic and hematologic function after coronary artery bypass grafting. Ann Thorac Surg 1999; 67: 1547-55.

Grigore AM, Mathew J, Grocott H, et al. Prospective randomized trial of normothermic versus hypothermic cardiopulmonary bypass on cognitive function after coronary artery bypass graft surgery. Anesthesiology 2001; 95: 1110-9.

Shiozaki T, Sugimoto H, Taneda $M$, et al. Effect of mild hypothermia on uncontrollable intracranial hypertension after severe head injury. J Neurosurg 1993; 79 : 363-8.

Marion DW, Penrod LE, Kelsey SF, et al. Treatment of traumatic brain injury with moderate hypothermia. N Engl J Med 1997; 336: 540-6.

Clifton GL, Miller ER, Choi SC, et al. Lack of effect of induction of hypothermia after acute brain injury. $\mathrm{N}$ Engl J Med 2001; 344: 556-63.

The Hypothermia after Cardiac Arrest Study Group. Mild therapeutic hypothermia to improve the neurologic outcome after cardiac arrest. N Engl J Med 2002; 346: 549-56.

Bernard SA, Jones BM, Horne MK. Clinical trial of induced hypothermia in comatose survivors of out-of-hospital 
cardiac arrest. Ann Emerg Med 1997; 30: 146-53.

Hindman BJ, Todd MM, Gelb AW, et al. Mild hypothermia

as a protective therapy during intracranial aneurysm

surgery: a randomized prospective pilot trial.

Neurosurgery 1999; 44: 23-32; discussion 32-3. 